\title{
Application of a proteomic approach to identify proteins associated with primary graft non-function after liver transplantation
}

\author{
OSKAR KORNASIEWICZ ${ }^{1 *}$, KAMIL BOJARCZUK $^{2 *}$, MAREK BUGAJSKI $^{2}$, \\ JAKUB GOLAB $^{2,3}$ and MAREK KRAWCZYK ${ }^{1}$
}

\author{
${ }^{1}$ Chair and Department of General, Transplant and Liver Surgery; ${ }^{2}$ Department of Immunology, \\ Center of Biostructure Research, Medical University of Warsaw, 02-097 Warsaw; ${ }^{3}$ Institute of \\ Physical Chemistry, Polish Academy of Sciences, Department 3, Warsaw, Poland
}

Received March 15, 2012; Accepted May 11, 2012

DOI: 10.3892/ijmm.2012.1062

\begin{abstract}
Primary graft non-function (PNF) is a rare, lifethreatening complication of liver transplantation. Increasing use of extended criteria donor pools and high-risk recipients seem to influence the incidence of PNF. Primary failure is associated with high patient morbidity and inferior graft survival. The only available treatment for PNF is emergency hepatic retransplantation, which is also correlated with significant morbidity and mortality. Therefore, researchers are working to identify risk factors of diagnostic value to prevent PNF. The current study attempted to explore liver proteomic patterns in patients with PNF. Using two-dimensional gel electrophoresis and liquid chromatography-mass spectrometry (LC-MS), we
\end{abstract}

Correspondence to: Professor Jakub Golab, Department of Immunology, Center of Biostructure Research, The Medical University of Warsaw, Banacha 1A, F building, 02-097 Warsaw, Poland

E-mail: jakub.golab@wum.edu.pl

Professor Marek Krawczyk, Chair and Department of General, Transplant and Liver Surgery, Medical University of Warsaw, Banacha 1A, 02-097 Warsaw, Poland

E-mail: marek.krawczyk@wum.edu.pl

*Contributed equally

Abbreviations: PNF, primary graft non-function; LC-MS, liquid chromatography-mass spectrometry; ICU, intensive care unit; UNOS, United Network for Organ Sharing; HAT, hepatic artery thrombosis; INR, international normalized ratio; AST, aspartate aminotransferase; ELTR, European Liver Transplant Registry; ECD, extended criteria donors; CIT, cold ischemia time; 2D-PAGE, two-dimensional polyacrylamide gel electrophoresis; IEF, isoelectric focusing; FL, functional liver; NCBInr, NCBI non-redundant human genome database; $\mathrm{I} / \mathrm{R}$, ischemia/reperfusion

Key words: orthotopic liver transplantation, primary liver graft failure, proteomics, 2D electrophoresis compared liver protein homogenates from 3 patients with PNF to those obtained from 6 healthy liver samples to identify potential new biomarkers of PNF. Our comparisons revealed 21 proteins with differential expression (13 upregulated and 8 downregulated). Most of these proteins are involved in energy metabolism, lipid metabolism, peptide cleavage, cell differentiation, and apoptosis. Although none of these proteins appeared more than once in separate analyses, this preliminary study shows that two-dimensional gel electrophoresis and LC-MS may allow identification of characteristic proteins to be used as biomarkers of a life-threatening complication of liver transplantation. Larger-scale analyses could improve patient care by finding suitable prognostic and therapeutic options. These data represent the first global proteomic approach to study PNF.

\section{Introduction}

Overall survival after liver transplantation has significantly improved in recent years. However, early graft failure remains a serious concern with high morbidity and mortality. Primary graft non-function (PNF) is a rare, but serious condition, of unknown pathophysiology developing in 2-6\% cases following liver transplantation (1-3). Although PNF was discovered more than 25 years ago, a clear consensus regarding the definition of PNF has not been reached (4). The United Network for Organ Sharing (UNOS) defines PNF as an irreversible graft function requiring emergency liver replacement within the first 10 days of liver transplantation. It is characterized by an aspartate aminotransferase (AST) of $\geq 5,000 \mathrm{UI} / 1$, international normalized ratio (INR) of $\geq 3.0$, and acidosis.

Other researchers have proposed a variety of definitions for graft non-function. Silberhumer et al suggested 4 grades of initial graft dysfunction, where PNF was defined as a clinical patient status requiring retransplantation within 7 days of primary transplantation (5). A further definition of PNF was presented by the European Liver Transplant Registry (ELTR), where PNF was restricted to retransplantation requirement within 1 month (30 days) of primary transplantation. Yet another interpretation was proposed by Amin et al, who 
altered the definition used by ELTR to 'a non-functioning graft within 1 month of receiving liver from the deceased donor' (6).

It is difficult to assess the incidence of PNF as it depends on the definition used. According to Kemmer et al, the incidence of PNF is similar in Europe and USA (7). The analysis conducted by Burroughs et al (8) on the data obtained from the ELTR (9) showed the incidence of PNF to be $6 \%$ and $3 \%$, where the cut-off time was 90 days and 30 days, respectively. Using the analysis of the 30-day time period, Kemmer et al demonstrated that although the utilization of extended criteria donors (ECD) over the last years had increased, the incidence of PNF has decreased (7).

A failing graft has been shown to lead to multi-organ instability, especially renal hemodynamic failure or generalized sepsis (10). Liver transplantation is the only treatment of choice for non-functioning grafts (11). The decision for retransplantation is mainly driven by clinical judgment and experience, partly because of the variance in the definitions of PNF. High perioperative recipient mortality is associated with the lack of instant availability of new grafts. In such cases, rescue hepatectomy has been proposed as a treatment, which aims to improve patient conditions while awaiting a retransplantation $(12,13)$. A large percentage of patients pass away because of systemic complications, which lead to multi-organ failure before a new graft becomes available.

The risk factors associated with PNF are related to the organ donor as well as to the organ recipient (14). In many studies, a donor over the age of 40 was associated with a significantly higher risk of graft failure $(15,16)$. In addition, hepatic steatosis of graft has also become a major issue, and susceptibility to steatosis increases with donor age, history of obesity, dyslipidemia, and diabetes. Macrovesicular steatosis is considered one of the most important risk factors for graft non-function (17). Other donor-related risk factors include $>5$-day stay in the intensive care unit (ICU), warm ischemia time of $>40 \mathrm{~min}$, cold ischemia time (CIT) of $>10 \mathrm{~h}$, and hypernatremia (18-21). In addition, research studies are constantly adding new risk factors and questioning the significance of the old risk factors for PNF (22-24).

Recent shortage in the available donor livers has led to the development of ECD (25-27). These include donors over the age of 70 , as well as donors with a history of hepatitis $\mathrm{C}$, inactive hepatitis $\mathrm{B}$, or liver steatosis. ECD carry a high risk of PNF (28); consequently, a decreased survival has been observed among high-risk recipients receiving organs from ECD (29).

Currently, the only effective strategy of prevention against primary graft non-function is donor and recipient selection according to known risk factors which does not fully prevent PNF. Little is known about the pathway of metabolic events leading to PNF, although interrupted microcirculation may play an important role (30). In contrast to previous studies focusing on factors involved in the event of PNF we hypothesized that a proteomic approach may lead to a better understanding of the biological aspect of graft non-function. Our 10-year experience of 1,000 transplantations at the Department of General, Transplant and Liver Surgery, (Medical University of Warsaw) support the relevance of this topic in patient survival.

In this study, we have used a proteomic approach to identify the proteins associated with PNF following liver transplantation. Our goal was to identify biomarkers that could be measured before liver transplantation to predict the grafts that will be susceptible to non-function. Clinical measurement of these biomarkers, in donors could potentially reduce the likelihood of PNF occurrence.

\section{Materials and methods}

Patient selection. Liver fragments were collected from 96 consecutive liver transplantations. All 96 pairs of samples were subjected to proteomic examination. Follow-up of the patients revealed that 3 of them (3.1\%) developed PNF within 10 days of transplantation (Table I). All other potential risk factors (HAT, acute rejection) were excluded in these 3 cases, where the grafts were considered to meet the criteria of PNF according to the UNOS criteria. All recipients received liver grafts from ECD, defined in our study as heart beating deceased donors over the age of 60 , with steatosis of $>30 \%$, $>5$-day stay in the ICU, CIT of $>8 \mathrm{~h}$, or $\left[\mathrm{Na}^{+}\right]$of $>170 \mathrm{mmol} / 1$ at least at one point in time. In each case, the following data were collected: cause of death, CIT, warm ischemia time, and post-reperfusion syndrome. Two patients were listed for, and subsequently underwent emergency retransplantation. The overall mortality in this group was $100 \%$. As a control group, we used 6 samples from donors with optimal grafts, who recovered after liver transplantation with a good synthetic liver function. On the basis of clinical, biochemical, and radiological parameters, the grafts were considered as either a normal/optimal or a suboptimal/EDC. After transplantation, clinical follow-up was carried out, and all the data were stored in a database. To minimize individual variability, we compared PNF and healthy liver protein lysates in 3 different sets, as described below.

This study was approved by the independent Ethics Committee of Warsaw Medical University in accordance with the ethics guidelines of the 'World Medical Association Declaration of Helsinki - Ethical Principles for Medical Research Involving Human Subjects' adopted by the 18th WMA General Assembly, Helsinki, Finland. Donor retrieval was performed under Polish law of organ donation supervised by the Polish Transplant Coordinating Centre 'Poltransplant' (www.poltransplant.org.pl). All recipients enrolled in this study provided written informed consent.

Sample preparation for protein extraction. Tissues were snapfrozen in nitrogen and stored at $-80^{\circ} \mathrm{C}$ until use. The frozen liver specimens (100-200 mg) were crushed to a fine powder by a ceramic mortar in liquid nitrogen. The fine powder was suspended in $1 \mathrm{ml}$ of rehydratation/sample buffer 1 (RSB1) containing $7 \mathrm{M}$ urea, $2 \mathrm{M}$ thiourea, 1\% ASB-14, $40 \mathrm{mM}$ Tris-Base, and trace bromophenol blue. Samples were then sonicated and centrifuged at $16,000 \mathrm{rcf}$ for $30 \mathrm{~min}$ at $20^{\circ} \mathrm{C}$. The supernatants were stored at $-80^{\circ} \mathrm{C}$ for further analyses. Protein disulfide bonds were reduced and alkylated using the ReadyPrep $^{\text {TM }}$ Reduction-Alkylation kit (Bio-Rad, Hercules, CA, USA), according to the manufacturer's protocol. Impure protein lysates were cleaned using the ReadyPrep ${ }^{\mathrm{TM}}$ cleanup kit (Bio-Rad), according to the manufacturer's protocol. Protein concentrations were measured by a modified Lowry assay using the RC DC Protein Assay (Bio-Rad). 
Two-dimensional polyacrylamide gel electrophoresis $(2 D-P A G E)$. Each $100 \mu \mathrm{g}$ of protein sample to be processed by isoelectric focusing (IEF) using PROTEAN ${ }^{\circledR}$ IEF cell (Bio-Rad) was diluted to a final volume of $500 \mu 1$ with the rehydratation/sample buffer $1^{+}$(RSB1 enriched with $2 \mathrm{mM}$ TBP and 0.2\% Bio-Lyte ${ }^{\circledR}$ 3/10 Ampholytes). The precast IPG strips ( $\mathrm{pH} 3-10$, linear $\mathrm{pH}$ gradient, 24-cm long), used for the first dimension, were actively rehydrated at $50 \mathrm{~V}$ and loaded with the sample at $20^{\circ} \mathrm{C}$ for $12 \mathrm{~h}$ in mineral oil. During the pause after rehydration, paper wicks soaked in ultrapure water were placed between each electrode and IPG strip. IEF was immediately initiated according to the following protocol: $10,000 \mathrm{~V}$ for $70,000 \mathrm{Vh}$, and then, at $500 \mathrm{~V}$. Strips were then equilibrated once for 30 min with gentle shaking in an equilibration solution containing $6 \mathrm{M}$ urea, $2 \%$ SDS, $0.375 \mathrm{M}$ Tris-HCl pH 8.8, and $20 \%$ glycerol. Separation by protein molecular mass was performed in a ProteanPlus ${ }^{\mathrm{TM}}$ Dodeca Cell (Bio-Rad) on homogeneous 10\%, 1-mm-thick polyacrylamide gels. Briefly, equilibrated IPG strips were placed onto gels and overlaid with ReadyPrep ${ }^{\mathrm{TM}}$ overlay agarose to remove any residual air bubbles from between the IPG strip and gel, and to add a trace of bromophenol blue for electrophoresis control. Second dimension SDS-PAGE was carried out according to the following protocol: $50 \mathrm{~V}$ for $5 \mathrm{~min}, 100 \mathrm{~V}$ for $10 \mathrm{~min}, 150 \mathrm{~V}$ for $15 \mathrm{~min}, 200 \mathrm{~V}$ for $20 \mathrm{~min}$, and $250 \mathrm{~V}$ until the blue dye reached the bottom of each gel. Immediately after electrophoresis, separated protein spots were visualized using Silver Stain Plus kit (Bio-Rad), according to the manufacturer's protocol.

Gel scanning and image analysis. Stained 2D gels were scanned and analyzed to compare matching spots. Gels were scanned using a Calibrated Densitometer (GS-800; Bio-Rad). Gel images were processed for spot detection, background subtraction, and matching by using the Quantity One and PDQuest software (Bio-Rad). For image analysis, the proteomic pattern of the control healthy liver was used as a reference pattern, and the primary non-function liver protein patterns were matched to this reference pattern. Spots were considered to be differentially expressed if they were either present in a different amount or absent in comparison with the reference gel. A quantitative difference was determined when the normalized total volume values differed significantly $(p<0.01$, Student's t-test). The ratio of expression intensity of PNF to that of the control of $\geq 2$ and $\leq 0.5$ were set as thresholds indicating significant change. Protein spots selected by 2D-PAGE were excised from the gels, washed with $1 \mathrm{ml}$ of ultrapure water, and identified by liquid chromatography followed by mass spectrometry (LC-MS). Peptide mass fingerprinting was performed with the MASCOT engine (Matrix Science, UK) against NCBI's non-redundant human genome database (NCBInr). The criteria for protein identification were based on probability-based MOWSE scoring algorithm with a 95\% confidence level in MASCOT.

\section{Results}

Proteome differential expression in functional and PNF livers. Total liver proteins were extracted from PNF and healthy samples, and separated by 2D-PAGE using IPG strips with

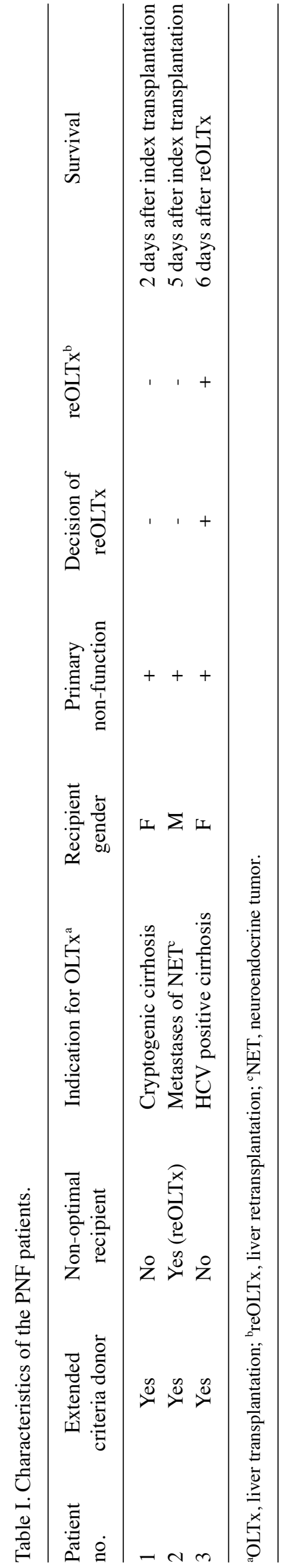


A

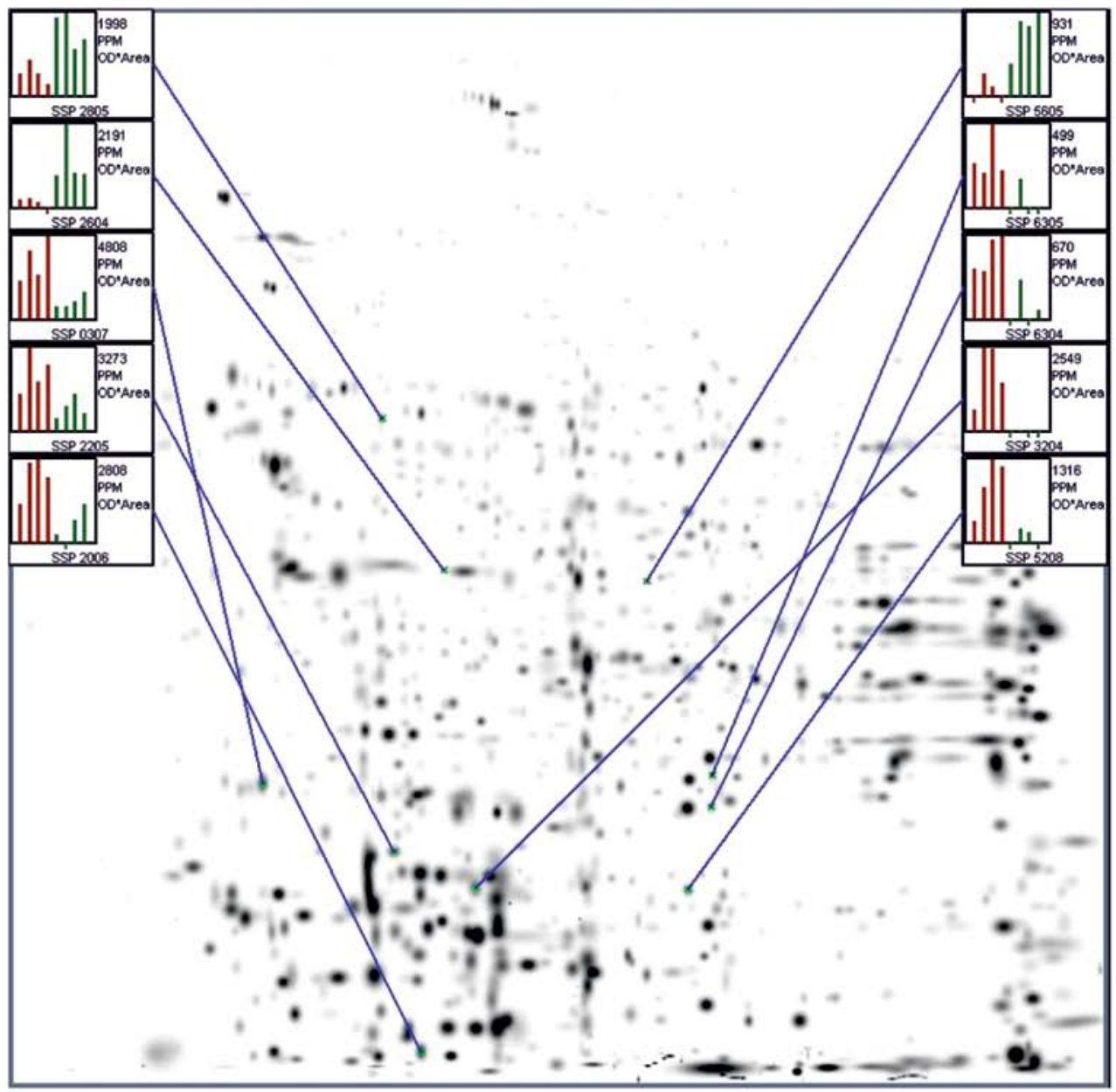

B

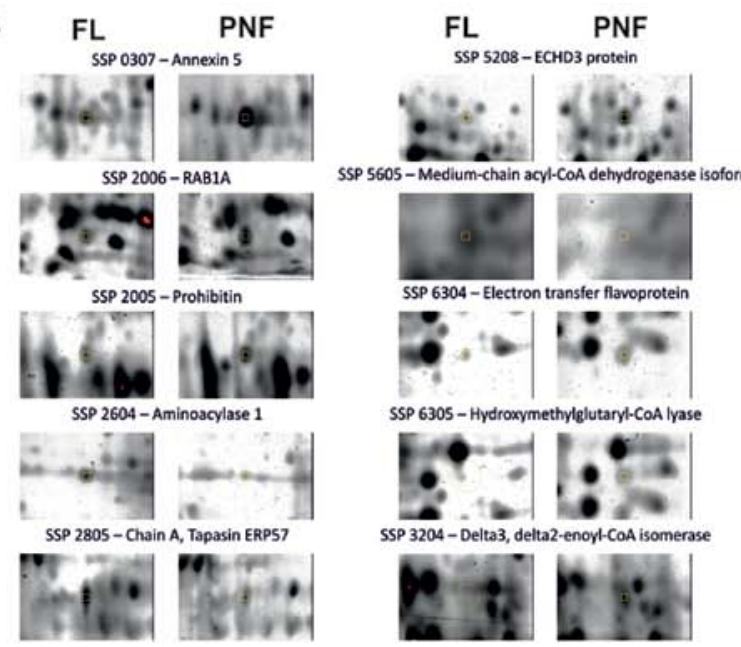

Figure 1. Representative 2D protein patterns of tandemly analyzed liver samples from functional liver (FL) and primary graft non-function (PNF) patients (first comparison pair). Sample preparation and 2D analysis were performed as described in the Materials and methods section. Proteins (100 $\mu \mathrm{g}$ ) were separated using linear IPG strips (pH 3-10) and 10\% SDS-PAGE, and were detected by silver staining. (A) Master gel shows complete view of liver proteins separated by 2D-PAGE. Insets indicate the differences between FL and PNF. (B) Higher magnification images of proteins analyzed by liquid chromatography, followed by mass spectrometry. Identified proteins are listed in Tables II and III.

a linear $\mathrm{pH}$ gradient ( $\mathrm{pH} 3-10)$. The proteins were resolved in homogeneous $10 \%$ acrylamide gels in the second dimension. After processing the 2D-PAGE gels of PNF livers and paired healthy tissues, well-resolved gels were obtained with the silver-stained spots both sharply focused and widely distributed along pH 3-10. Using Quantity One and PDQuest 
A

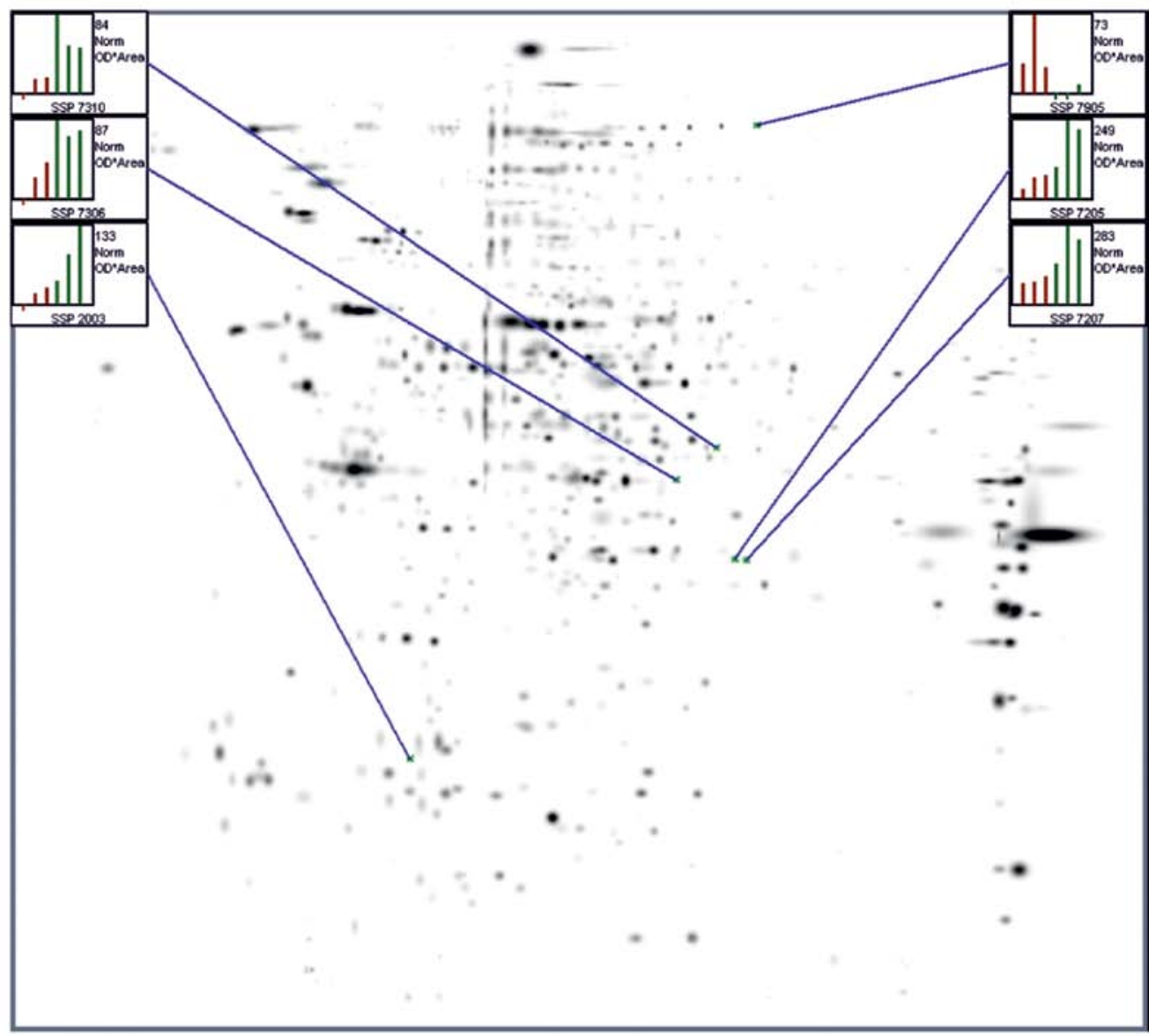

B

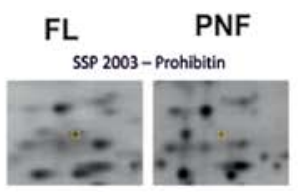

$\mathrm{FL}$

PNF

SSP 7306 - NADP-dependent isocitrate dehyfrogenase

SSP 7205 - Aldolase B

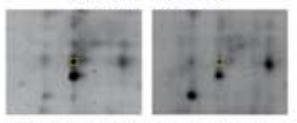

SSP 7207 - Ornithine transcarbamylase
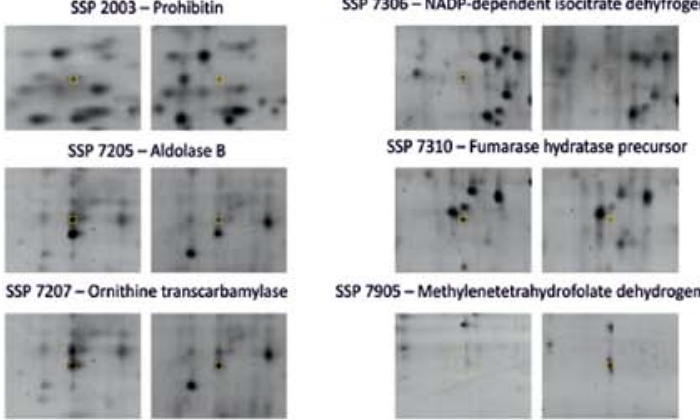

SSP 7905 - Methylenetetrahydrofolate dehydrogenase 1

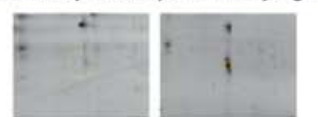

Figure 2. Representative 2D protein patterns of tandemly analyzed liver samples from functional liver (FL) and primary graft non-function (PNF) patients (second comparison pair). Sample preparation and 2D analysis were performed as described in the Materials and methods section. Proteins (100 $\mu$ g) were separated using linear IPG strips (pH 3-10) and 10\% SDS-PAGE, and were detected by silver staining. (A) Master gel shows complete view of liver proteins separated by 2D-PAGE. Insets indicate the differences between FL and PNF. (B) Higher magnification images of proteins analyzed by liquid chromatography, followed by mass spectrometry. Identified proteins are listed in Tables II and III.

software, about 1,300 spots per gel were detected, and their numbers were automatically determined.

Analysis of differentially expressed proteins. Samples processed under identical conditions can be compared by 2D-PAGE. Therefore, tandem experiments were performed to compare pairs of samples prepared from functional livers (FL), those that performed adequately in subsequent followup assessments after transplantations, to that of PNF livers, that failed to regain normal synthetic function after transplantation.

Representative 2D-PAGE maps obtained from 3 independent separations are shown in Figs. 1, 2 and 3. For reliable analysis of protein expression, 2D gel maps were constructed and analyzed in replicates. In the first comparison, both PNF and FL samples were separated on 4 independent gels. The master gel, an average of 4 replicate runs of the gels, calculated and averaged in silico, was selected as the reference gel. The 
A

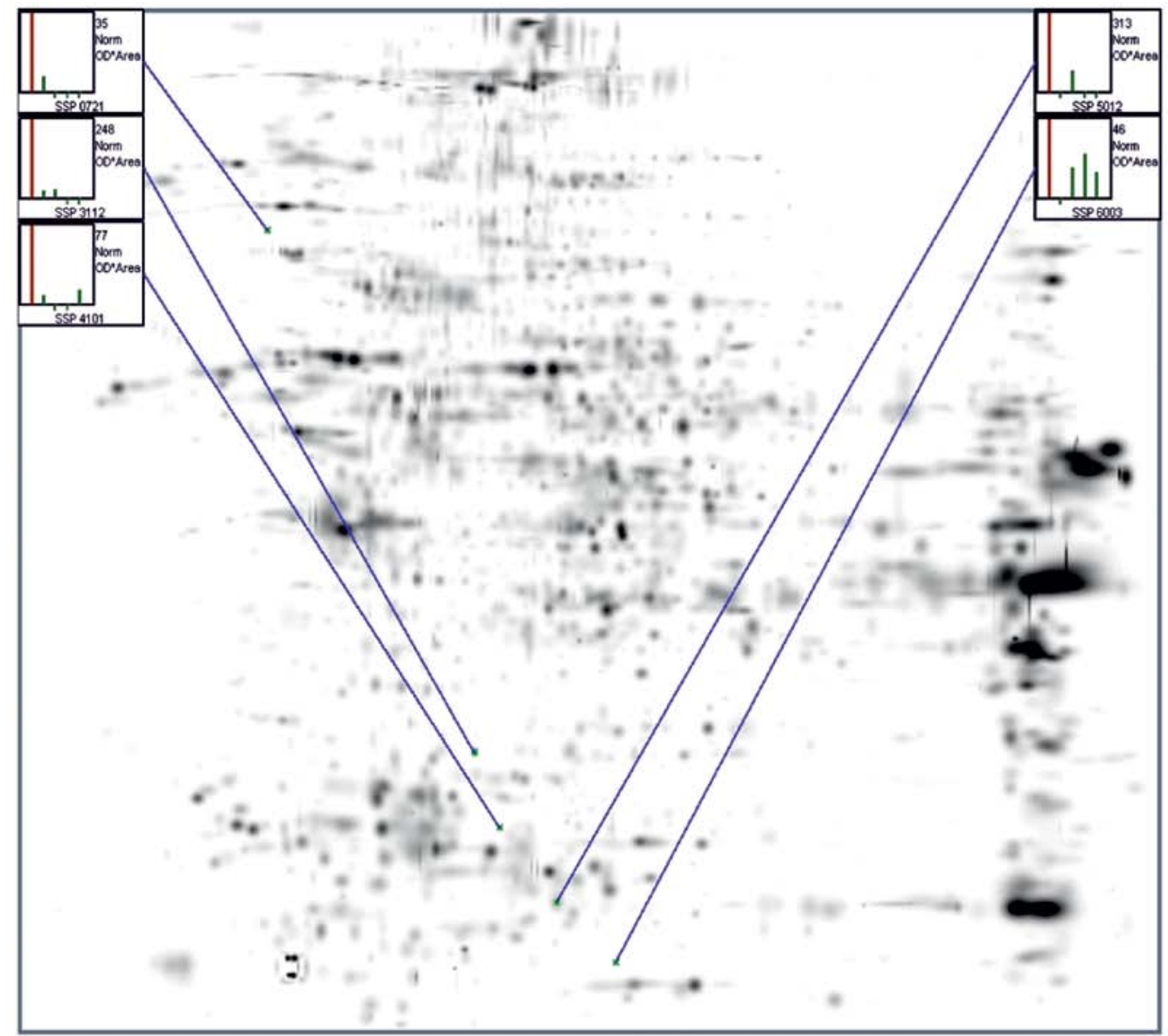

B

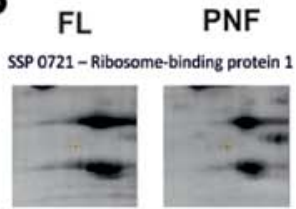

SSP 3112 - Caspase 14 prcursor

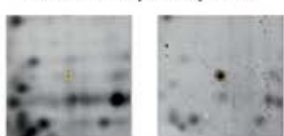

SSP 4101 - Proteasome subunit alpha

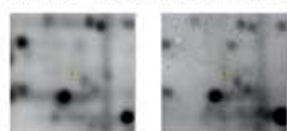

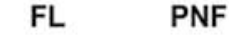

SSP 5012 - Macropain subunit iota

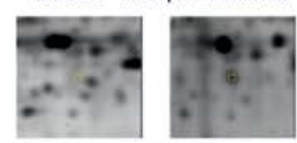

SSP 6003 - Proteasome subunit bet

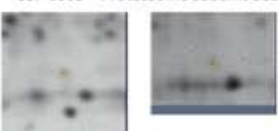

Figure 3. Representative 2D protein patterns of tandemly analyzed liver samples from functional liver (FL) and primary graft non-function (PNF) patients (third comparison pair. Sample preparation and 2D analysis were performed as described in the Materials and methods section. Proteins (100 $\mu \mathrm{g}$ ) were separated using linear IPG strips (pH 3-10) and 10\% SDS-PAGE, and were detected by silver staining. (A) Master gel shows complete view of liver proteins separated by 2D-PAGE. Insets indicate the differences between FL and PNF. (B) Higher magnification images of proteins analyzed by liquid chromatography, followed by mass spectrometry. Identified proteins are listed in Tables II and III.

Student's t-test showed that the volume of 10 protein spots was significantly altered in these gels (Fig. 1, p<0.01). In the second separation, all the samples were separated on 2 independent gels, and the master gel was selected as the reference gel.The Student's t-test showed that the volume of 6 protein spots was significantly altered on these gels (Fig. $2, \mathrm{p}<0.01$ ). In the third separation, one PNF sample was separated on 4 independent gels, while 4 healthy samples were separated on 1 gel each, and the master gel was selected as the reference gel. The student's t-test showed that the volume of the 5 protein spots was significantly altered in the gels (Fig. 3, p<0.01). Altogether, using the procedures described above, we found 21 differential 2D-PAGE spots in the PNF livers: 13 that were upregulated and 8 that were downregulated, compared to the control livers. 
Table II. Mass spectrometry-based identification of the 2D-PAGE protein spots differently expressed in primary non-functional human livers.

\begin{tabular}{|c|c|c|c|c|c|}
\hline $\begin{array}{l}\text { Protein } \\
\text { number }\end{array}$ & $\begin{array}{c}\text { Spot } \\
\text { number }\end{array}$ & Protein name & Score ${ }^{a}$ & $\begin{array}{l}\text { Theoretical pI; } \\
\operatorname{Mr}(\mathrm{kDa})\end{array}$ & PNF vs. FL \\
\hline 1 & 0307 & Annexin 5 & 148 & $4.94 ; 36$ & Upregulation \\
\hline 2 & 2006 & $\begin{array}{l}\text { RAB } 1 \mathrm{~A} \text {, member Ras oncogene } \\
\text { family isoform } 1\end{array}$ & 335 & $5.93 ; 23$ & Upregulation \\
\hline 3 & 2205 & Prohibitin & 362 & $5.57 ; 29.8$ & Upregulation \\
\hline 4 & 2604 & Aminoacylase 1 & 169 & $5.77 ; 46$ & Downregulation \\
\hline 5 & 2805 & Chain A, tapasin ERP57 heterodimer & 432 & $5.61 ; 54.5$ & Downregulation \\
\hline 6 & 3204 & $\Delta^{3}, \Delta^{2}$-enoyl-CoA isomerase & 416 & $6.39 ; 29.7$ & Upregulation \\
\hline 7 & 5208 & ECHDC3 protein & 129 & $8.81 ; 31.5$ & Upregulation \\
\hline 8 & 5605 & $\begin{array}{l}\text { Medium-chain acyl-CoA dehydrogenase } \\
\text { isoform } \alpha \text { precursor }\end{array}$ & 124 & $8.61 ; 47$ & Downregulation \\
\hline 9 & 6304 & Electron transfer flavoprotein & 393 & $8.62 ; 35.4$ & Upregulation \\
\hline 10 & 6305 & Hydroxymethylglutaryl-CoA lyase & 137 & $7.01 ; 32.6$ & Upregulation \\
\hline 11 & 2003 & Prohibitin & 595 & $5.57 ; 29.8$ & Downregulation \\
\hline 12 & 7205 & Aldolase B & 456 & $8.00 ; 40$ & Downregulation \\
\hline 13 & 7207 & Ornithine transcarbamylase & 183 & $8.84 ; 40$ & Downregulation \\
\hline 14 & 7306 & NADP-dependent isocitrate dehydrogenase & 146 & $6.34 ; 46.9$ & Downregulation \\
\hline 15 & 7310 & Fumarate hydratase precursor & 396 & $8.85 ; 54.8$ & Downregulation \\
\hline 16 & 7905 & $\begin{array}{l}\mathrm{NADP}^{+} \text {-dependent methylenetetrahydro- } \\
\text { folate dehydrogenase } 1\end{array}$ & 189 & $6.75 ; 102.2$ & Upregulation \\
\hline 17 & 0721 & Ribosome-binding protein 1 & 630 & $5.45 ; 109$ & Upregulation \\
\hline 18 & 3112 & Caspase 14 precursor & 137 & $5.44 ; 28$ & Upregulation \\
\hline 19 & 4101 & Proteasome subunit $\alpha$ type- 3 isoform 1 & 108 & 5,$19 ; 28.6$ & Upregulation \\
\hline 20 & 5012 & Macropain subunit ı & 98 & $5.58 ; 25.1$ & Upregulation \\
\hline 21 & 6003 & Proteasome subunit $\beta$ type- 2 isoform 1 & 142 & $6.51 ; 23$ & Upregulation \\
\hline
\end{tabular}

${ }^{a}$ Individual ions scores $>53$ indicate identity or extensive homology $(\mathrm{p}<0.05)$. Protein scores are derived from ions scores as a non-probabilistic basis for ranking protein hits.

Identification of proteins by LC-MS. All of the 21 differentially expressed protein spots were excised from the gels and identified by liquid chromatography followed by mass spectrometry (LC-MS). Peptide mass fingerprinting performed with the MASCOT engine against NCBInr identified the proteins listed in Table II. In Table III, the 21 proteins shown, are grouped according to function and localization.

\section{Discussion}

The prevalence of PNF has remained steady over the past 2 decades, since the syndrome has been discovered. Nonetheless, increasing incidence of PNF has already been documented and can be expected to continue an increasing number of ECD donors are accepted for transplantation (7). Regular surveillance of high-risk grafts is carefully carried out each time a donor is accepted by a transplant center for recovery. Despite all efforts, even an experienced retrieval team and accepted means of preventive treatment may not protect the recipient from PNF in every case. Often, the combination of risk factors associated with each graft can be responsible for the problem. When evaluated independently, their impact on PNF is not obvious, but when they coexist, the graft dysfunction is clinically more significant. Therefore, we believe that the results of a proteomic approach will provide valuable novel data to evaluate grafts for PNF.

In our experience, $>50 \%$ of the donors fulfill the criteria of ECD. All patients included in the study developed graft non-function following transplantation of grafts received from ECD. The currently extended donor criteria used by the Department of General, Transplant and Liver Surgery are generally consistent with the literature. The donor was considered ECD if at least 2 of the criteria were fulfilled. If steatosis is encountered during organ recovery, the other variables of ECD graft are then considered.

Although liver retransplantation is the only definitive treatment for PNF, it carries a high mortality rate (2). However, successful retransplantations with long-term follow-up showed that the patient and graft survival rates after retransplantation following PNF were not different compared to retransplantation for alternative causes (31). In contrast, Yoo et al showed inferior results of retransplantation for PNF (2). Nevertheless, in spite of high perioperative mortality, we are in favor of emergency retransplantation. Therefore, 2 of 3 patients were listed 


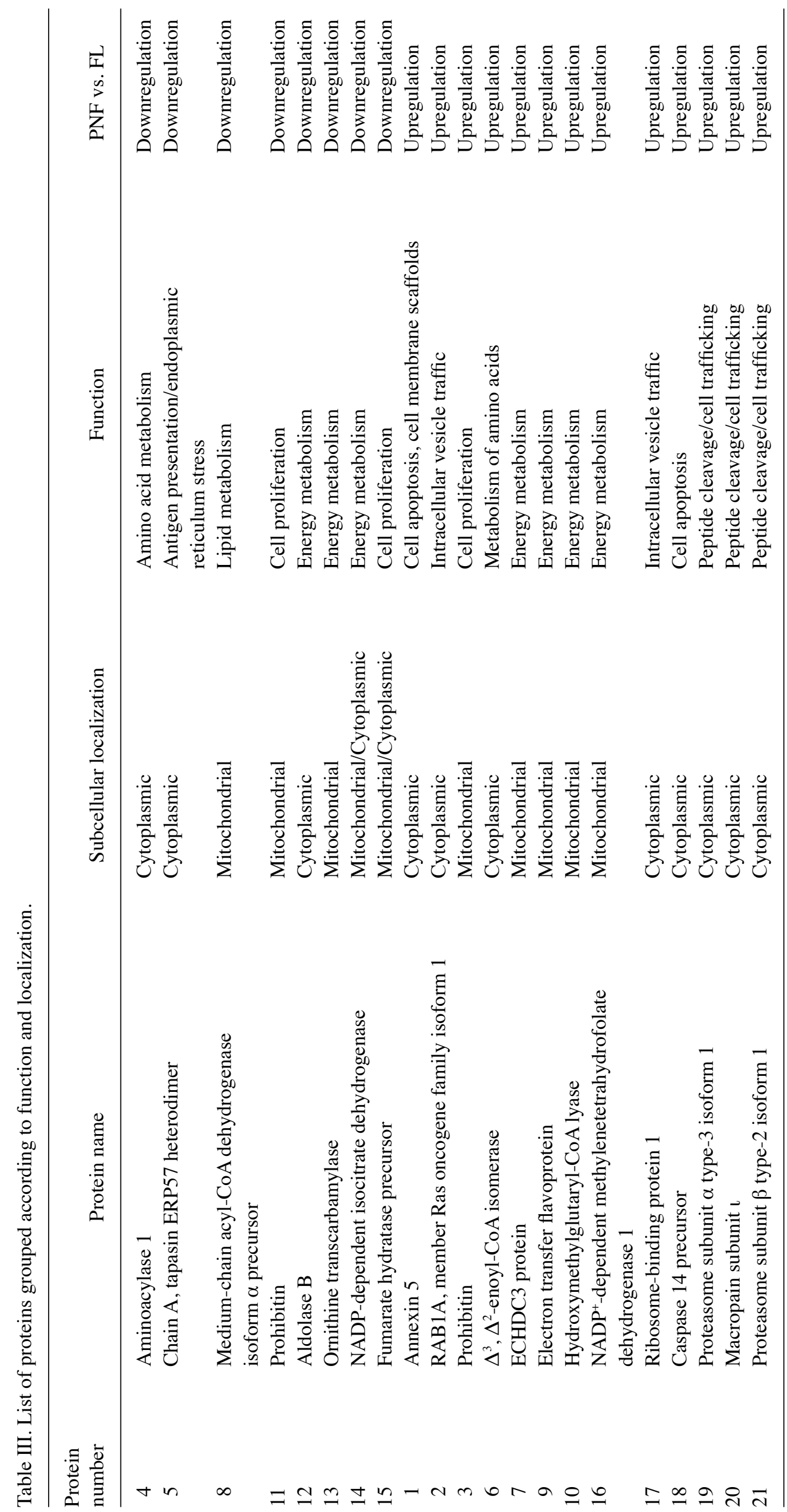


for reOLTx. In our group, the survival was $0 \%$. In all cases, multi-organ failure was considered the cause of death. The general critical condition of the remaining 1 recipient made it impossible to offer retransplantation as a treatment. One patient passed away because of systemic complications, which led to multi-organ failure before a new graft was available. One patient passed away 6 days after liver retransplanation reOLTx.

To the best of our knowledge, this is the first attempt to utilize a proteomic approach in the study of PNF in order to identify novel predictive biomarkers. To do this, the protein expression profile of the liver grafts transplanted to recipients who developed PNF were compared to those with optimal liver grafts, which showed normal primary function after transplantation. Proteomics-based technologies can identify and quantify novel proteins that can function as biomarkers of the presence or severity of a disease state. In general, human liver proteome profiling is quite challenging. A minority of proteins, including albumin, immunoglobulins, transferrin, and fibrinogen, are highly abundant, and typically constitute $>90 \%$ of the total protein mass. However, the low abundant proteins are most likely to be biologically relevant as the markers of a disease state. In this study, we present the liver proteome analysis of patients with PNF and functional liver after transplantation. We identified changes in 21 proteins (13 upregulated and 8 downregulated) when comparing the samples from the PNF grafts to those from the grafts with normal function after transplantation.

Tables II and III list the proteins that were either upregulated or downregulated. The characteristic functions of the differentially expressed proteins can be classified into two major categories: one category is proteins associated with mitochondrial oxidative phosphorylation, while the other category is proteins essential for the ATP-dependent turnover of proteins. Among the upregulated proteins, 4 are related to energy metabolism: ECHDC3 protein, electron transfer flavoprotein, hydroxymethylglutaryl-CoA lyase, and NADP ${ }^{+}$ dependent ehydrogenase 1 .

Only 2 of the upregulated proteins are associated with cell apoptosis: Annexin 5 and caspase 14 precursor. This observation indicates that hepatocyte apoptosis is not a major factor for PNF. One may also speculate that even a suboptimal graft is not prone to dysfunction prior to harvesting simply on the basis cell death pathways. It may also imply that events leading to PNF are multifactorial and are rather metabolic in origin and thereby potentially reversible. Indirect proof of that hypothesis is a relative rarity of the PNF in contrast to the increasing number of ECD.

Only one among the upregulated proteins, an electron transfer flavoprotein, is associated with cellular protection against oxidative stress (ischemia/reperfusion [I/R] injury). Vascotto et al reported the upregulation of flavoproteins when they compared the changes in liver proteome profile upon reperfusion (32), but this is the only reported occurrence of such a finding. This may mean that the grafts were not subjected to a major ischemic stress, which might impair the metabolic function after reperfusion.

The 8 proteins that were downregulated in the PNF livers are all involved in various metabolic pathways, mostly in energy and energy substrate metabolism. Vascotto et al also reported an increase in the hepatic levels of aldolase $\mathrm{B}$, a protein responsible for energy metabolism, after reperfusion (32). In our data, aldolase B was downregulated in only one case, indirectly suggesting the lack of a major ischemic insult to the graft before donation. Considering that tissues were collected from deceased donors, we can hypothesize that the alterations in these protein levels are not directly related to ischemic tissue responses.

None of the proteins appeared more than once in separate analyses. The absence of similar proteomic patterns among the cases studied may indicate that PNF is a heterogeneous multifactor phenomenon, and that a graft is not deemed to primary dysfunction on a proteomic basis. A relatively small number of upregulated and downregulated proteins in our study may also indicate that PNF grafts were in a stable metabolic homeostasis before retrieval and not much different from optimal grafts. Additional factors may have also influenced the outcome. These factors may be linked with cold and warm ischemia time, liver perfusion, or possibly, post-reperfusion syndrome. We may conclude that it is not the graft itself, but other factors leading to $\mathrm{I} / \mathrm{R}$ syndrome that function as the determinants of primary dysfunction (32). Therefore, our data may be useful in determining the likelihood of I/R syndrome rather than primary graft non-function. At present, there is not enough data to support the notion that a proteomic study alone is a useful tool for diagnosing primary PNF.

Our study has demonstrated that grafts developing PNF demonstrate modification of the liver proteome. Considering the complexity of the problem, which can be influenced by many external and internal factors, finding one specific pattern of biomarkers will be difficult. Therefore, rather than focusing on the expression of each individual protein, we focused our attention on the global aspects of variations in protein expression with respect to a functional response. The majority of protein expression changes were noted among proteins associated with energy metabolism. The other major groups of proteins found in PNF were proteins involved in ATP-dependent turnover of proteins and lipids, as well as cell trafficking control. These changes were noted only in single cases, so they may represent an alteration of their normal involvement in metabolic pathways typical for the liver, the site of amino acids and lipid metabolism. In our opinion, the alteration of these protein levels was not directly related to the ischemic tissue response or other insult to the potential graft.

Our study has certain limitations. The definition of PNF used in our study was provided for graft non-function occurring within 10 days of transplantation. In addition, the study analyzed only a small number of cases. We think that an increased sample size could potentially alter the results. Another limitation of the study was the difficulty involved in collecting a similar group of samples in a controlled and repetitive manner. Quite a few variations were beyond the control of the researchers. For example, the grafts were recovered and implanted by different teams of surgeons. However, the research protocol was very strict, and a single surgeon performed the entire procedure of sample freezing and preparation for further research. There are some variables in the 2D-PAGE procedure that could not be overcome. For example, the temperature at which silver staining is performed has tremendous impact on the intensity of stained protein spots 
(33). Another considerable problem that occurs during the preparation of protein samples is the lack of protein-loading control. To address these technical problems, all tandem analyses were performed under strictly controlled conditions, including simultaneous staining and multiple independent measurements of sample protein concentration.

In summary, this study identified 21 proteins differentially expressed in a PNF graft sample compared to normal liver grafts. The individual protein associations identified in the proteomic study validate the technique. The data also led us to hypothesize that a unique profile for PNF may not be possible because of many donor-associated factors. A further explanation of the problem is that PNF is a complex phenomenon. Therefore, in our opinion, it would be worthwhile to search for a global aspect of a unique proteomic profile that would be clinically relevant to the discovery of diagnostic, prognostic, and therapeutic biomarkers to advance the knowledge and treatment of PNF.

\section{Acknowledgements}

This study was supported by grants N 40306732/3612 (to M.K.) from the Polish Ministry of Science. J.G. is supported by the European Union within European Regional Development Fund through Innovative Economy grant POIG.01.01.02-00-008/08. J.G. is a recipient of the Mistrz Award from the Foundation for Polish Science. J.G. is a member of TEAM Programme co-financed by the Foundation for Polish Science and the EU European Regional Development Fund.

\section{References}

1. Ploeg RJ, D'Alessandro AM, Knechtle SJ, et al: Risk factors for primary dysfunction after liver transplantation-a multivariate analysis. Transplantation 55: 807-813, 1993.

2. Yoo HY, Maheshwari A and Thuluvath PJ: Retransplantation of liver: primary graft nonfunction and hepatitis $C$ virus are associated with worse outcome. Liver Transpl 9: 897-904, 2003.

3. Uemura T, Randall HB, Sanchez EQ, et al: Liver retransplantation for primary nonfunction: analysis of a 20 -year single-center experience. Liver Transpl 13: 227-233, 2007.

4. Shaw BW Jr, Gordon RD, Iwatsuki S and Starzl TE: Retransplantation of the liver. Semin Liver Dis 5: 394-401, 1985.

5. Silberhumer GR, Pokorny H, Hetz H, et al: Combination of extended donor criteria and changes in the Model for End-Stage Liver Disease score predict patient survival and primary dysfunction in liver transplantation: a retrospective analysis. Transplantation 83: 588-592, 2007.

6. Amin MG, Wolf MP, TenBrook JA Jr, et al: Expanded criteria donor grafts for deceased donor liver transplantation under the MELD system: a decision analysis. Liver Transpl 10: 1468-1475, 2004.

7. Kemmer N, Secic M, Zacharias V, Kaiser T and Neff GW: Long-term analysis of primary nonfunction in liver transplant recipients. Transplant Proc 39: 1477-1480, 2007.

8. Burroughs AK, Sabin CA, Rolles K, et al: 3-month and 12-month mortality after first liver transplant in adults in Europe: predictive models for outcome. Lancet 367: 225-232, 2006.

9. Adam R, McMaster P, O'Grady JG, et al: Evolution of liver transplantation in Europe: report of the European Liver Transplant Registry. Liver Transpl 9: 1231-1243, 2003.

10. Pitre J, Soubrane O, Dousset B, et al: How valid is emergency liver transplantation for acute liver necrosis in patients with multiple-organ failure? Liver Transpl Surg 2: 1-7, 1996.
11. Doyle HR, Morelli F, McMichael J, et al: Hepatic retransplantation - an analysis of risk factors associated with outcome. Transplantation 61: 1499-1505, 1996.

12. Oldhafer KJ, Bornscheuer A, Frühauf NR, et al: Rescue hepatectomy for initial graft non-function after liver transplantation. Transplantation 67: 1024-1028, 1999.

13. So SK, Barteau JA, Perdrizet GA and Marsh JW: Successful retransplantation after a 48-hour anhepatic state. Transplant Proc 25: 1962-1963, 1993.

14. Chen H, Peng CH, Shen BY, et al: Multi-factor analysis of initial poor graft function after orthotopic liver transplantation. Hepatobiliary Pancreat Dis Int 6: 141-146, 2007.

15. Lake JR, Shorr JS, Steffen BJ, Chu AH, Gordon RD and Wiesner RH: Differential effects of donor age in liver transplant recipients infected with hepatitis $B$, hepatitis $C$ and without viral hepatitis. Am J Transplant 5: 549-557, 2005.

16. Feng S, Goodrich NP, Bragg-Gresham JL, et al: Characteristics associated with liver graft failure: the concept of a donor risk index. Am J Transplant 6: 783-790, 2006.

17. Clavien PA, Selzner M, Rüdiger HA, et al: A prospective randomized study in 100 consecutive patients undergoing major liver resection with versus without ischemic preconditioning. Ann Surg 238: 843-852, 2003.

18. Strasberg SM, Howard TK, Molmenti EP and Hertl M: Selecting the donor liver: risk factors for poor function after orthotopic liver transplantation. Hepatology 20: 829-838, 1994.

19. Totsuka E, Dodson F, Urakami A, et al: Influence of high donor serum sodium levels on early postoperative graft function in human liver transplantation: effect of correction of donor hypernatremia. Liver Transpl Surg 5: 421-428, 1999.

20. Yersiz H, Shaked A, Olthoff K, et al: Correlation between donor age and the pattern of liver graft recovery after transplantation. Transplantation 60: 790-794, 1995.

21. Cameron AM, Ghobrial RM, Yersiz H, et al: Optimal utilization of donor grafts with extended criteria: a single-center experience in over 1000 liver transplants. Ann Surg 243: 748-755, 2006.

22. Johnson SR, Alexopoulos S, Curry M and Hanto DW: Primary nonfunction (PNF) in the MELD era: An SRTR database analysis. Am J Transplant 7: 1003-1009, 2007.

23. Busquets J, Xiol X, Figueras J, et al: The impact of donor age on liver transplantation: influence of donor age on early liver function and on subsequent patient and graft survival. Transplantation 71: 1765-1771, 2001.

24. Washburn WK, Johnson LB, Lewis WD and Jenkins RL: Graft function and outcome of older ( $>$ or $=60$ years) donor livers. Transplantation 61: 1062-1066, 1996.

25. Pokorny H, Gruenberger T, Soliman T, Rockenschaub S, Längle F and Steininger R: Organ survival after primary dysfunction of liver grafts in clinical orthotopic liver transplantation. Transpl Int 13 (Suppl 1): S154-S157, 2000.

26. Müllhaupt B, Dimitroulis D, Gerlach JT and Clavien PA: Hot topics in liver transplantation: organ allocation - extended criteria donor - living donor liver transplantation. J Hepatol 48 (Suppl 1): S58-S67, 2008

27. Chung HY, Chan SC, Lo CM and Fan ST: Strategies for widening liver donor pool. Asian J Surg 33: 63-69, 2010.

28. Busuttil RW and Tanaka K: The utility of marginal donors in liver transplantation. Liver Transpl 9: 651-663, 2003.

29. Gastaca M: Extended criteria donors in liver transplantation: adapting donor quality and recipient. Transplant Proc 41: 975-979, 2009.

30. Hatsugai K, Ohkohchi N, Fukumori T, Akamatsu Y and Satomi S: Mechanism of primary graft non-function in a rat model for fatty liver transplantation. Transpl Int 13 (Suppl 1): S583-S590, 2000.

31. Azoulay D, Linhares MM, Huguet E, et al: Decision for retransplantation of the liver: an experience- and cost-based analysis. Ann Surg 236: 713-721, 2002.

32. Vascotto C, Cesaratto L, D'Ambrosio C, et al: Proteomic analysis of liver tissues subjected to early ischemia/reperfusion injury during human orthotopic liver transplantation. Proteomics 6: 3455-3465, 2006.

33. Chevallet M, Luche $\mathrm{S}$ and Rabilloud T: Silver staining of proteins in polyacrylamide gels. Nat Protoc 1: 1852-1858, 2006. 